https://doi.org/10.15407/ujpe64.8.750

W. SCHÄFER, ${ }^{1}$ I. BABIARZ, ${ }^{1}$ A. CISEK,${ }^{2}$ A. SZCZUREK ${ }^{1}$

${ }^{1}$ Institute of Nuclear Physics, Polish Academy of Sciences

(152, Ul. Radzikowskiego, PL-31-142 Kraków, Poland; e-mail: wolfgang.schafer@ifj.edu.pl)

2 Faculty of Mathematics and Natural Sciences, University of Rzeszów,

(1, Ul. Pigonia, PL-35-310 Rzeszów, Poland)

\title{
RECENT RESULTS ON INCLUSIVE QUARKONIUM PAIR PRODUCTION IN PROTON-PROTON COLLISIONS
}

\begin{abstract}
Recently, there has been much interest in the pair production of charmonia. One of the main motivations behind these studies is that the production of quarkonium pairs is expected to receive an important contribution from the double parton scattering (DPS) production mode. A large effective cross-section $\sigma_{\mathrm{eff}}$ is found from the empirical analysis of the $J / \psi$-pair production - about a factor 2.5 smaller than the usually accepted $\sigma_{\mathrm{eff}}=15 \mathrm{mb}$. Here, we present the recent results of our calculations of the $\chi_{c}$ pair production, mainly in the single parton scattering (SPS) mode. An important feature is that the single-gluon exchange mechanism can to some extent mimic the behavior of the DPS production.
\end{abstract}

Keywords: perturbative QCD, quarkonia, multiparton processes.

\section{Introduction}

The production of $J / \psi$-pairs has been suggested as a probe of the double-parton scattering (DPS) processes [1]. More generally, the DPS production mode is expected to be especially important in the charm sector [2]. Therefore, recently, there has been much interest in the quarkonium pair production in proton-proton collisions also from the experimental side. Among others, the cross-sections for the production of $J / \psi$-pairs were measured at the Tevatron [3] and the LHC [4-7].

A number of puzzles remain with these data, however. For example, the single parton scattering (SPS) leading order of $\mathcal{O}\left(\alpha_{S}^{4}\right)$ (see, e.g., $[8,9]$ ) does not describe well all the kinematic distributions in the case of the ATLAS and CMS data. Especially, when the rapidity distance $\Delta y$ between two $J / \psi$ mesons is large, it falls short of experimental data. If one ascribes the whole discrepancy to DPS processes, the normalization of DPS comes out a factor $\sim 2.5$ larger than in other hard processes. It is still an open issue at the moment whether this points to a nonuniversality of DPS effects or whether there are additional

(C) W. SCHÄFER, I. BABIARZ, A. CISEK, A. SZCZUREK, 2019 single parton scattering mechanisms not taken into account up to now.

This problem motivated our recent studies of the $\chi_{c}$-pair production in the $k_{T}$-factorization [10] and of the production of $\chi_{c}$-pairs associated with a gluon (jet) in the collinear factorization [11]. We summarize these works in this contribution.

\section{Production of $\chi_{c}$-Pairs}

In the standard hard scattering approach, the crosssection of the production of a pair of quarkonia $a, b$ is calculated from a convolution of parton densities with a parton-level cross-section (see the left diagram in Fig. 1). However, at high energies, favored by a rise of the gluon distribution at small $x$, there is a sizable contribution from processes in which two or more hard processes proceed in the same proton-proton collision (see the right diagram in Fig. 1).

One commonly assumes the factorized ansatz for the production cross- section in the DPS mode:

$$
\begin{aligned}
& \frac{d \sigma_{\mathrm{DPS}}(p p \rightarrow a b X)}{d y_{a} d y_{b} d^{2} \mathbf{p}_{a T} d^{2} \mathbf{p}_{b T}}= \\
& =\frac{1}{1+\delta_{a b}} \frac{1}{\sigma_{\mathrm{eff}}} \frac{d \sigma(p p \rightarrow a X)}{d y_{a} d^{2} \mathbf{p}_{a T}} \frac{d \sigma(p p \rightarrow b X)}{d y_{b} d^{2} \mathbf{p}_{b T}} .
\end{aligned}
$$

ISSN 2071-0194. Ukr. J. Phys. 2019. Vol. 64, No. 8 

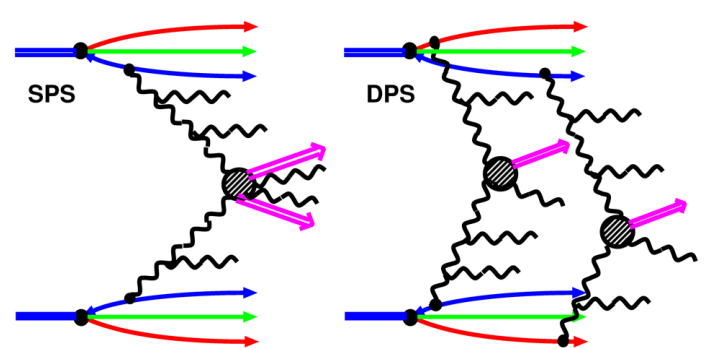

Fig. 1. Sketch of the single parton scattering (SPS) and double parton scattering (DPS) production modes

The DPS cross-section is written as a product of the inclusive single-particle spectra, and the cross-section is normalized by the "effective cross-section" $\sigma_{\text {eff }}$. The latter is not the cross-section for a specific process the real parameter is rather its inverse, which is related in the simplest model to the overlap of parton densities in the transverse plane, $t_{N}(\mathbf{b})$ :

$$
\begin{aligned}
& \frac{1}{\sigma_{\mathrm{eff}}}=\int d^{2} \mathbf{b} T_{N N}^{2}(\mathbf{b}), \\
& T_{N N}(\mathbf{b})=\int d^{2} \mathbf{s} t_{N}(\mathbf{s}) t_{N}(\mathbf{b}-\mathbf{s}) .
\end{aligned}
$$

The salient features of DPS are obvious from Eq. (1). Important for us is the observation that each of the single particle spectra is a fairly broad function of $y_{a, b}$. Thus, the DPS distribution in rapidity distance $\Delta y=y_{b}-y_{a}$ will be very broad as well. As far as the effective cross-section is concerned, it is usually taken in the ballpark of $\sigma_{\text {eff }}=15 \mathrm{mb}$, which is within the line of a fair amount of hard processes, see, e.g., a table in [5].

In the case of $J / \psi$-pair production, the lowest-order "box-diagram" mechanism suggests a very clean separation of SPS versus DPS modes. Indeed, the explicit calculations performed in the $k_{T}$-factorization [9] show that the $J / \psi$-pair distribution is sharply peaked around $\Delta y=0$.

A main point of this presentation is the fact that the situation looks completely different in the case of production of a pair of $\chi_{c}$ mesons. Indeed, the $\chi_{c J}$ states, which come in three different spins $J=0,1,2$ have positive $C$-parity and thus couple to two gluons in a color singlet state. Hence, the mechanism of Fig. 2 with the $t$-channel exchange of a single gluon is possible. It is well understood that it will lead to a $g g \rightarrow \chi \chi$ cross-section independent of the cm-energy in the high-energy limit. The matrix element for this

ISSN 2071-0194. Ukr. J. Phys. 2019. Vol. 64, No. 8

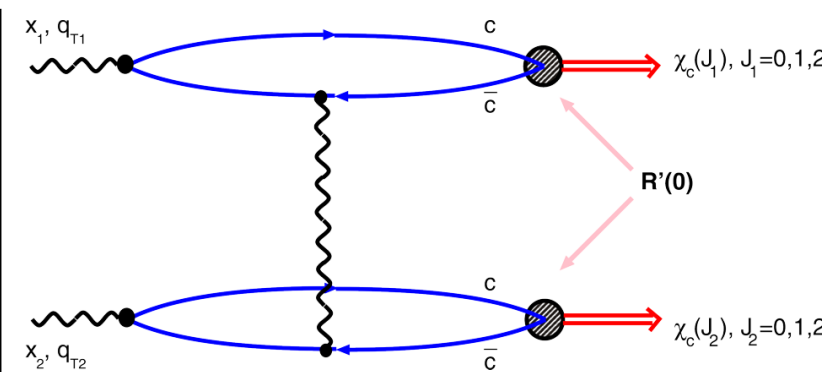

Fig. 2. Gluon $t$-channel exchange mechanism for the production of $\chi_{c} \chi_{c}$ pairs

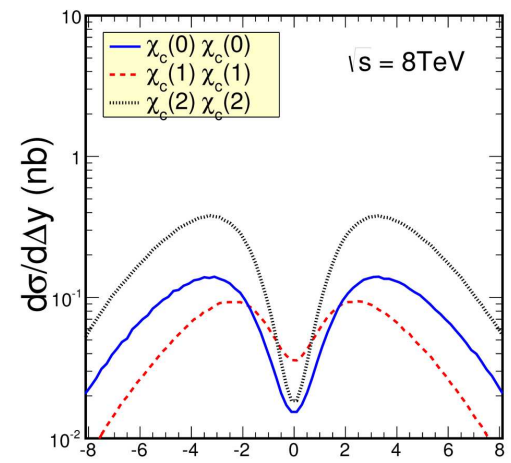

$\Delta y$

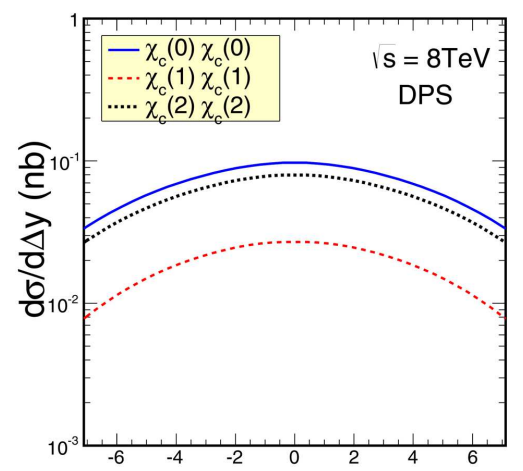

$\Delta y$

Fig. 3. Distribution of $\chi_{c}$-pairs in the rapidity difference between mesons. Top panel: SPS mode, lower panel: DPS mode

process thus puts no penalty on a large rapidity distance $\Delta y$ between the $\chi_{c}$-mesons.

The relevant amplitudes can be obtained from effective $g^{*} g^{*} \rightarrow \chi_{c J}$ vertices for the fusion of two spacelike off-shell gluons. These have been obtained in Ref. [10] for all possible spin-states of the $\chi_{c}$ family. We also performed calculations in the $k_{T^{-}}$ factorization including the transverse momenta of incoming gluons. In the upper panel of Fig. 3, we 

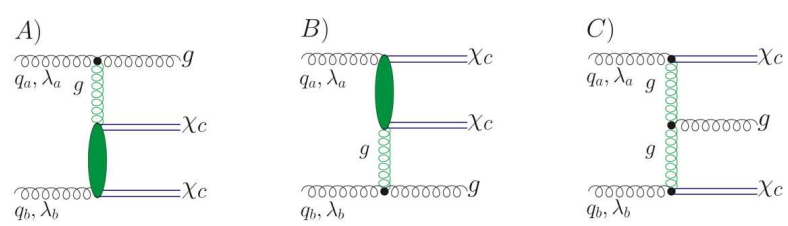

Fig. 4. Feynman diagrams for the production of a $\chi_{c}$-pair associated with a gluon
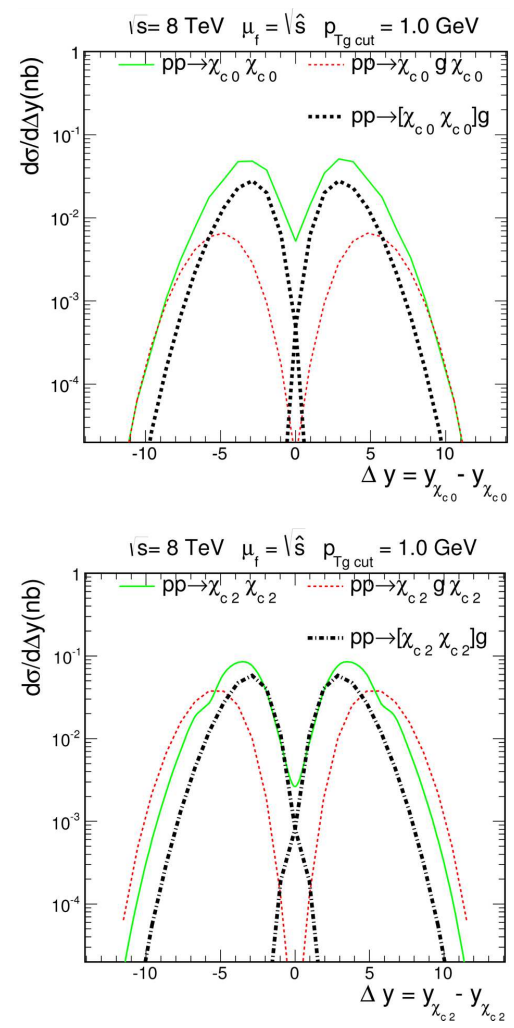

Fig. 5. Distribution in rapidity between $\chi_{0}$ mesons (top panel) and $\chi_{c 2}$ mesons for the following different processes: Born-level production of $\chi_{c}$-pairs, production of $\chi_{c}$ pairs with a leading gluon, and production of $\chi_{c}$-pairs with a central gluon

show the distribution in rapidity distance $\Delta y$ between mesons. Note that we only show, as an example, the production of pairs of identical mesons, the full array of all possible combinations can be found in Ref. [10]. In the lower panel of Fig. 3, we show distributions in $\Delta y$ for the DPS mode, by using $\sigma_{\text {eff }}=15 \mathrm{mb}$. We see that these distributions are very broad and in the same ballpark as the SPS contribution. Of course, there is no minimum at $\Delta y=0$ for the DPS distributions. Thus, we observe rather similar distributions in $\Delta y$ for single and double par- ton scattering productions of different $\chi_{c}$-quarkonia states. This shows that both contributions must be included in the analysis of future data on the $\chi_{c J_{i}} \chi_{c J_{j}}$ production. Now, one would observe that the large rapidity distance between mesons means a large phase space for the emission of additional gluons. To investigate this situation, we studied the associated production of $\chi_{c}$ pairs with a gluon in the standard collinear factorization in Ref. [11]. There are two main contributions shown in the diagrams of Fig. 4: first, the emission of a "leading gluon", where the gluon jet carries a large fraction of the momentum carried by one of the incoming gluons, and, second, the production of "central" gluons, which are emitted in the rapidity space between two mesons with a large difference in rapidity from either one. Some distributions, again in rapidity distance $\Delta y$ between mesons, are shown in Fig. 5. The production of leading gluons adds to the Born-result to recover the $k_{T}$-factorization result, while the production of central gluons gives rise to an about $20 \%$ enhancement of the cross-section. Here, one may think of $\alpha_{S} \Delta y$ as a large parameter which could be resummed in the future using the BFKL formalism.

\section{Conclusions}

The pair production of quarkonia is a topic that still poses puzzles to theorists. The quantitative understanding of DPS contributions requires not only a reliable formalism for its calculation but also a good understanding of SPS processes that can show a similar behavior as DPS in many kinematic variables.

For the theoretically simplest case, the production of $\chi_{c}$-pairs, we have shown that the cross-sections for different combinations of $\chi_{c}$ quarkonia, the SPS and DPS cross-sections, are of the similar size, and both involve very broad distributions in the rapidity distance $\Delta y$.

We have also shown that an enhancement of the pair production cross-section for $\chi_{c}$-pairs can be expected from the higher-order corrections, due to the large phase space of the gluon emission.

However, it turns out that the feed-down from $\chi$ pairs into the $J / \psi$-pair channel does not resolve the discrepancy between different determinations of $\sigma_{\text {eff }}$.

It might be necessary to look deeper into the fundamentals of the DPS theory (see, e.g., [13]) to understand the peculiar behavior of the charmonium pair production.

ISSN 2071-0194. Ukr. J. Phys. 2019. Vol. 64, No. 8 
The participation of W.S. in the conference New Trends in High Energy Physics 2019 has been partially supported by the Polish Academy of Sciences.

1. C. H. Kom, A. Kulesza, W. J. Stirling. Pair production of $J / \psi$ as a probe of double parton scattering at LHCb. Phys. Rev. Lett. 107, 082002 (2011).

2. M. Łuszczak, R. Maciuła, A. Szczurek. Production of two $c \bar{c}$ pairs in double-parton scattering. Phys. Rev. D 85, 094034 (2012).

3. V.M. Abazov et al., [D0 Collaboration]. Observation and studies of double $J / \psi$ production at the Tevatron. Phys. Rev. D 90, 111101 (2014).

4. V. Khachatryan et al., [CMS Collaboration]. Measurement of prompt $J / \psi$ pair production in pp collisions at $\sqrt{s}=$ $=7$ Tev. JHEP 1409, 094 (2014).

5. M. Aaboud et al., [ATLAS Collaboration]. Measurement of the prompt $\mathrm{J} / \psi$ pair production cross-section in pp collisions at $\sqrt{s}=8 \mathrm{TeV}$ with the ATLAS detector. Eur. Phys. J. C 77, 76 (2017).

6. R. Aaij et al., [LHCb Collaboration]. Observation of $J / \psi$ pair production in $p p$ collisions at $\sqrt{s}=7 \mathrm{TeV}$. Phys. Lett. $B$ 707, 52 (2012).

7. R. Aaij et al., [LHCb Collaboration]. Measurement of the $\mathrm{J} / \psi$ pair production cross-section in pp collisions at $\sqrt{s}=$ $=13 \mathrm{TeV}$. JHEP 1706, 047 (2017); Erratum: JHEP 1710, 068 (2017).

8. S.P. Baranov. Pair production of $J / \psi$ mesons in the $k_{t^{-}}$ factorization approach. Phys. Rev. D 84, 054012 (2011).

9. S.P. Baranov, A.M. Snigirev, N.P. Zotov, A. Szczurek, W. Schäfer. Interparticle correlations in the production of $J / \psi$ pairs in proton-proton collisions. Phys. Rev. D 87, 034035 (2013).
10. A. Cisek, W. Schäfer, A. Szczurek. Production of $\chi_{c}$ pairs with large rapidity separation in $k_{T}$ factorization. Phys. Rev. D 97, 114018 (2018).

11. I. Babiarz, W. Schäfer, A. Szczurek. Associated production of $\chi_{c}$ pairs with a gluon in the collinear-factorization approach. Phys. Rev. D 99, 074014 (2019).

12. W. Schäfer. Production of quarkonium pairs in highenergy proton-proton collisions. EPJ Web Conf. 199, 01021 (2019).

13. J. R. Gaunt, R. Maciuła, A. Szczurek. Conventional versus single-ladder-splitting contributions to double parton scattering production of two quarkonia, two Higgs bosons and $c \bar{c} c \bar{c}$. Phys. Rev. D 90, 054017 (2014).

Received 26.01.18

В. Шефер, І. Бабяж, А. Чішек, А. Щурек

НОВІ РЕЗУЛЬТАТИ

ПРО ІНКЛЮЗИВНЕ НАРОДЖЕННЯ

ПАР КВАРКОНІУМУ

В ПРОТОН-ПРОТОННИХ ЗІТКНЕННЯХ

$\mathrm{P}$ е $з$ ю м е

Останнім часом спостерігається значний інтерес до процесів парного народження шармонія. Однією з причин інтересу є те, що продукування пар кварконіуму в значній мірі зумовлене подвійним розсіянням партонів (DPS). 3 емпіричного аналізу народження пар $J / \psi$ знайдено велике значення ефективного перерізу $\sigma_{\text {eff }}=15$ мб. Ми представляємо нові результати наших розрахунків продукування пар $\chi_{c}$ в моді одинарного партонного розсіяння (SPS). Важливим моментом є те, що однопіонний обмін в деякій мірі може симулювати ефект подвійного партонного обміну (DPE). 